Artículo de investigación

Cuestiones de filosofía

ISSN: 0123-5095

Vol. 2 - No. 19

Julio - dciembre, año 2016

pp. $105-126$

\title{
Foucault: una lectura en clave discontinua en el periodo arqueológico*
}

\author{
Foucault: a discontinuous reading \\ on the archaeological period
}

Osman Daniel Choque-Aliaga*

Universidad de San Buenaventura

Colombia

Fecha de recepción: 21 de abril del 2016

Fecha de evaluación: 8 de Agosto del 2016

Fecha de aceptación: 21 de octubre de 2016

* Trabajo de investigación realizado durante el seminario en torno a Foucault realizado por la Maestría en Filosofía Contemporánea de la Universidad de san Buenaventura, sede Bogotá.

** Magíster en Filosofía Contemporánea, de la Universidad de san Buenaventura, sede Bogotá. Especialista en Filosofía Contemporánea, de la misma Universidad.junker.odca@gmail.com 


\title{
Resumen
}

Sin duda, el tema de la discontinuidad ha llegado a acaparar la atención de un buen número de investigadores que se dedican a reflexionar en torno a la filosofía de Foucault. Posar ahora la mirada en el tema de la discontinuidad promete abrir una veta nueva de análisis que, quizá, permita revalorar el alcance de los aportes filosóficos de este pensador francés. Para tal tarea, el artículo, en un primer momento, se acerca a la noción de historia en el pensamiento foucaultiano, para a partir de ahí estudiar el desenvolvimiento que dicha noción tiene con la discontinuidad; luego, se detiene a estudiar el método arqueológico, para desentrañar si es posible analizar el tema de la discontinuidad a la luz de la arqueología. ¿Será posible mirar el tema de la discontinuidad como un rasgo propio del pensamiento del filósofo francés? Responder esta pregunta es la tarea que este trabajo pretende llevar a fin.

Palabras clave: Arqueología, Discontinuidad, Filosofía, Foucault, Historia

\begin{abstract}
The topic of discontinuity is undoubtedly one of the most popular among a great deal of the researchers who devote themselves to reflect upon the philosophy of Foucault. Giving that kind of attention to the topic of discontinuity might open a whole new source of analysis, with the possibility of reevaluating the scope of Foucault's contributions to philosophy. With that purpose, this article will, at first, make an approach to the notion of history in Foucaultian thought, in order to study the way in which this notion is related to the concept of discontinuity. Consequently, this article makes a close study of the archeological method, to find out if an analysis of the topic of discontinuity using an archeological approach is viable. Would it be possible to understand the topic of discontinuity as a standard feature of the philosophy of this French thinker? The present work is meant to answer this question.
\end{abstract}

Key words: Archaeology, discontinuity, philosophy, Foucault, history. 


\section{INTRODUCCIÓN}

"Todo pensamiento es un pensamiento discontinuo".

G. Canguilhem

El tema de la discontinuidad ha llegado a ocupar un lugar muy importante en los estudios póstumos acerca del pensamiento de Foucault, como lo deja ver el número creciente de investigaciones que han hecho de esta cuestión uno de los hilos conductores para moverse en la obra del pensador francés (Morey, 2014; Deleuze, 1987; Castro, 1995; Revel, 2014). La atención creciente que los estudiosos de Foucault le dedican a este tema es, sin duda, un incentivo para intentar indagar por nuestros medios lo que hay en juego en esta temática, y establecer qué luces puede arrojar su tratamiento para una mejor comprensión del pensamiento foucaultiano. Ciertamente, el tema constituye una novedad, en la medida en que los tópicos que durante mucho tiempo han dominado la recepción de la obra de Foucault han sido la historia, el sujeto y el poder, sobre los cuales, como sabemos, se han hecho fecundos estudios que han sabido destacar la complejidad y profundidad de su pensamiento (p. ej., Ceballos, 1997; Le Blanc, 2008; Sauquillo, 2001). Posar ahora la mirada en el tema de la discontinuidad promete abrir una veta nueva de análisis que, quizá, permita revalorar el alcance de los aportes filosóficos del pensador francés. Pero, ¿de dónde surge el interés por este tema? y ¿qué es lo que convierte la pregunta por la discontinuidad en una línea de lectura promisoria para adentrarse en la obra de Foucault?

Ya desde el llamado periodo arqueológico de su trabajo, Foucault causaba inquietud entre sus lectores al oponerse claramente a una idea de historia entendida como acumulación progresiva y lineal de momentos que tenderían a sintetizarse en un telos último de sentido, $\mathrm{y}$, antes bien, describirla como atravesada de saltos que hacían imposible cualquier explicación causal o continua de los acontecimientos. Tal discontinuidad impedía conferir sentido alguno al decurso histórico. Es el caso de Las palabras y las cosas (1966), libro en el que la sucesión de una episteme a otra (de la edad clásica al renacimiento, y de esta a la modernidad) no admite ser vista como el paso progresivo de unas reglas discursivas a otras más eficaces para describir la realidad, sino que supone rupturas bruscas en las capas profundas que en cada época hacen posible el surgimiento de los discursos de verdad. Foucault reafirma esta idea cuando, más adelante, en el periodo llamado 
genealógico, adhiere a la visión de Nietzsche de la historia marcada por quiebres ocurridos por la lucha de fuerzas interpretativas en busca de dominio (Cfr. Foucault, 2000). Sin embargo, cabe preguntarse siel tema de la discontinuidad solo aparece en el marco de la reflexión de Foucault sobre la historia o si ocupa otros lugares en la obra del autor, si es apenas marginal o si constituye una vena por la que corre buena parte de su reflexión filosófica.

Edgardo Castro reconoce la importancia del tema al afirmar que:

Foucault no aborda la historia del conocimiento en términos de continuidad, sino de discontinuidad. Por ello, en lugar de manejarse en términos de evolución, de lo que anticipa y de lo que realiza, lo hace en términos de ruptura. Foucault se pregunta, de hecho, acerca de lo que hace posible en un momento dado que ciertas cosas hayan sido dichas. Las condiciones de posibilidad del saber son condiciones en el nivel de la simultaneidad (2004, p. 56).

Este pensador introduce el tema de la discontinuidad como una referencia importante en los textos de Foucault. Es verdad que Castro establece una relación clara entre la discontinuidad y el tratamiento que el filósofo francés hace de la historia, a contrapelo de una visión moderna en donde la historia tiene un rasgo fundamental: la continuidad, es decir, los acontecimientos se ven concatenados formando un resultado compacto, gradual y ordenado. En El vocabulario de Michel Foucault (2004), Castro se detiene de manera rápida en la noción de discontinuidad (término que según su conteo aparece unas 128 veces en el corpus foucaultiano, a diferencia, por ejemplo, del de historia, que figura 4687 veces). Esta comparación ya se nos insinúa interesante a la hora de destacar el interés por el tema de la discontinuidad, que, aunque discretamente, como nos lo demuestra Castro, ha capturado la mirada de los estudiosos de Foucault.

Por su parte, Judith Revel ha llevado a cabo una lectura de la obra de Foucault inspirándose en la discontinuidad como clave de lectura del escritor francés. "El tema de la discontinuidad, es decir, tanto el del rechazo de una representación continuista y lineal de la historia como, en términos generales, de los modelos epistemológicos que hacen de la continuidad una prenda de herencia, está en el núcleo del trabajo de Foucault" (2009, p. 49). Revel asume que la discontinuidad es una noción clave tanto en la crítica de Foucault a la noción de historia como a los modelos epistemológicos. Su atracción por la discontinuidad habría llevado a Foucault a dar un nuevo tratamiento a la noción de historia, así como a la noción de poder. 
A mi parecer, Revel no llega a describir su arquitectónica interna, es decir, no describe a fondo la manera como el tema de la discontinuidad da su forma al conjunto de la obra de Foucault; sin embargo, esta autora ofrece un aporte importante al no contentarse con señalar que la discontinuidad es un tema central tratado por Foucault, sino que ve en la discontinuidad un rasgo propio del pensamiento del filósofo francés. Algo así como que la discontinuidad, menos que objeto de estudio, es su método o forma de pensar. Entonces, cabe preguntar: ¿qué es la discontinuidad para Foucault?, ¿podría tomársela como una noción clave para entender el método de la arqueología o de la genealogía empleados por Foucault?, ¿cómo funcionaría o trabajaría ese método de la discontinuidad?, ¿explica la manera como Foucault establece los cortes históricos en sus análisis, asunto que levantó polémica en su momento?, ¿se advierte esta atención sobre la discontinuidad en la forma como trabaja y estudia un discurso, por ejemplo? y ¿se trata de un determinado y peculiar modo de pensar enraizado en todo el itinerario filosófico del pensador francés?

Las dos referencias anteriores nos demuestran que estamos ante un proyecto investigativo que puede determinar muchas características del pensamiento de Foucault; sobre todo, confirman que el tema de la discontinuidad merece atención especial. En lo que sigue dejaré de lado, por el momento, estas cuestiones, y me concentraré en identificar la manera cómo surge, en el periodo inicial del itinerario filosófico de Foucault (1962-1969), el tema de la discontinuidad, particularmente en el marco de sus reflexiones sobre la historia. Así, pues, en un primer momento se llevará a cabo un esbozo acerca de su concepción de la historia a partir de la obra Las palabras y las cosas, para luego relacionarla con la discontinuidad; en el segundo momento desplazaré la atención al método arqueológico, teniendo en cuenta que, como señalé, también en su tratamiento de este método se advierte una impronta del tema de la discontinuidad, y finalmente, en el último momento de este trabajo, se determinará si efectivamente la discontinuidad es un tema que recorre el pensamiento de Foucault a lo largo del periodo arqueológico y, sobre todo, se evaluará si, más que un tema, la discontinuidad en Foucault se refiere a un pensamiento que piensa discontinuamente.

\section{LA DISCONTINUIDAD EN EL ANÁLISIS HISTÓRICO}

Las palabras y las cosas está dividido en dos bloques. En el primero se encuentra el análisis de Las Meninas, de Velásquez; aquí Foucault describe la complejidad de miradas y posiciones en esa pintura, y deja entrever su camino de análisis y lo que buscará demostrar en los capítulos siguientes: cada periodo tiene su propia y 
peculiar manera de pensar las cosas; dicho de otra manera, cada periodo de la historia tiene sus propias condiciones de sacar a la superficie la verdad de sus discursos, aquello que después será aceptado o no. En el análisis acerca de las condiciones como surgen conocimientos, el pensador francés argumenta que estas cambian con el tiempo; son totalmente variables; se trata para Foucault de giros repentinos, rupturas y cambios de un periodo a otro. En la parte restante del primer bloque y en todo el segundo, Foucault se dedica a argumentar los cambios de la episteme que se dan en cada época, pasando por el renacimiento, por la época clásica y terminando su análisis en la época moderna. Entonces, dos cuestiones son centrales en Las palabras y las cosas: por un lado, la noción de episteme, y, por otro, la descripción de tres epistemes que se suceden entre los periodos de los siglos XVI y XIX.

Foucault habla, por otro lado, de arqueología (como dice el subtítulo de Las palabras y las cosas: Una arqueología de las ciencias humanas) para referirse al método con el cual aborda la historia de las ciencias humanas, ya que pretende revelar las formas epistemológicas que en una época determinada rigen la organización y aparición de todos los discursos científicos que pasan luego a formar parte de un conocimiento como tal, es decir, cierto estatuto de lo que conocemos hoy como ciencias humanas. En ese sentido, el pensador francés entiende las epistemes como las condiciones de posibilidad ocultas que intervinieron para la emergencia de tales discursos. Revelar estas formas epistemológicas es un método que se aparta del que empleaba la historia más convencional, deteniéndose Foucault en las manifestaciones de los discursos mismos y, más aún, alejándose de la historiografía científica. Ahora bien, las formas epistemológicas, o episteme, son la ciencia de aquello que determina los temas, los problemas, los debates intrínsecos de una época. En ese orden de ideas, lo que descubre Foucault es que la historia de los discursos de saber estaría caracterizada por una sucesión de epistemes que cada época histórica caracteriza por unas reglas internas. La episteme es única y propia y la que determina los discursos, limita las fronteras entre un discurso y otro, encauza las ideas de los saberes y la reflexión que ha sido posible hacer de ella. La episteme es, entonces, "el conjunto de relaciones capaces de unir, en una época dada, las prácticas discursivas que dan lugar a figuras epistemológicas, a ciencias y, eventualmente, a sistemas formalizados" (Foucault, 1969, p. 250). Y, ¿quién sabe todo ello?, ¿quién puede delimitar los alcances de una episteme?, ¿quién puede analizar la conciencia de un momento histórico? ¿quién puede diagnosticar la formación de una episteme entre varios siglos? Foucault responde: "la conciencia de la episteme es privilegio del arqueólogo" (Burgelin, Revault y 
Amiot, 1970, p. 151); por lo tanto, el arqueólogo que es Foucault nos ofrece un estudio de las epistemes de tres periodos: la episteme del renacimiento, de la época clásica y de la modernidad.

Ahora bien, Las palabras y las cosas, identifica la transformación de la racionalidad occidental entre los siglos XVI y XIX en su paso por las tres epistemes antes mencionadas. Lo que busca Foucault con dicho análisis es contrarrestar la idea de continuidad histórica, es decir, la idea de una historia acumulativa de acontecimientos históricos que discurre lineal y unidireccionalmente y que describe un progreso. La novedad que trae Las palabras y las cosas radica justamente en determinar que no existe una sola historia homogénea que recorriendo varios periodos encuentre y describa el progreso de un periodo al siguiente. No es un proceso que, abarcando las tres epistemes, describa un discurso horizontal que avanza de forma apacible y absoluta. Cuando Foucault muestra el proceso, la relación y la distinción de una episteme a otra no encuentra alguna explicación en el paso de una a otra, ya sea en una perspectiva progresiva o que vaya avanzando a paso lento hacia un mismo punto. La historia de los discursos de saber, la historia de las ciencias humanas, tema del que se ocupa Foucault, no tiene nada de una visión positivista por la que se quemarían etapas hacia un proceso de maduración y lucidez progresiva de la razón. Se trata de algo más complejo, pues, la cuestión es la siguiente: Las palabras y las cosas describe el paso por tres epistemes; en cada una hay una historia interna, la historia de una episteme; cada episteme se expresa en pensamientos y lenguaje; tres epistemes que se dividieron según dos grandes rupturas.

La episteme del Renacimiento tenía una estrecha relación con el lenguaje: "la naturaleza misma es un tejido ininterrumpido de palabras y de marcas, de relatos y de caracteres, de discursos y de formas" (Foucault, 1968, p. 47). El mundo, el lenguaje, el conocimiento y el saber estaban dominados en este periodo por la semejanza. En este periodo los saberes tenían tres canales de expresión: la oralidad, el comentario y la interpretación, siendo estas formas de comprender los saberes; sin embargo, en el siglo Xvi el lenguaje, según Foucault, no pudo tener contacto directo con la realidad: lo que ha llevado en este periodo a hablar de posibles "aproximaciones".

En la semejanza existen dos figuras que pretendieron acercar el saber a la realidad: el loco y el poeta. El loco, a partir de su locura, se acercó a la realidad, quiso comprenderla, penetrando cada vez más con sus movimientos, sus gestos hacia el mundo. De la misma manera, el poeta, con sus rimas y versos, pretendió acercarse 
y tener una experiencia plena con el momento presente. El Quijote hace suya la realidad y, sobre todo, elimina la distancia que han venido cargando el poeta y el loco. ¿Cómo lo hace? Por medio del lenguaje. El lenguaje en este periodo es el que habla, y cuanto más se hable, más cerca estará uno de acercarse a la realidad; la manifestación de la locuacidad, del habla mediante el lenguaje. El lenguaje de $E l$ Quijote fue la plenitud de la semejanza; semejanza con la realidad, con lo que se pudo saber acerca de la realidad, "las aventuras de Don Quijote trazan el límite: en ellas terminan los juegos antiguos de la semejanza y de los signos; allí se anudan nuevas relaciones. Don Quijote no es el hombre extravagante, sino más bien el peregrino meticuloso que se detiene en todas las marcas de la similitud" (Foucault, 1968, p. 53).

¿Quién es El Quijote? Para Foucault, fue el puente entre una episteme y la otra. Es el personaje del Quijote el que va a llevar la semejanza hasta el límite, "Don Quijote debe ser fiel a este libro en el que, de hecho, se ha convertido" (1968, p. 55), y con él se cierra la época del renacimiento; la nueva episteme de la época clásica será la representación, dejando de lado y rompiendo la línea que había trazado la primera.

De la época del Renacimiento a la época Clásica sucede un cambio: el lenguaje ya no habla, ahora analiza la episteme del orden y la clasificación. "Ya no se efectúan aproximaciones, se discierne" (Burgelin et al., 1970, p. 39). De la semejanza, pasando por la representación, se dio paso a hablar del orden: "la posibilidad de establecer entre las cosas, incluso en las no mensurables, una sucesión ordenada" (1968, p. 71). Se trató de la voluntad de captar lo mecánico y calculable en la naturaleza; es una aplicación de la conocida mathesis universalis. es decir, que una lógica, un tipo de mirada, una forma de análisis, una manera de hablar rigió y se paseó en todo este periodo: el orden, y, de la mano de él, los dominios empíricos fueron guiados por la mecánica y la geometría. AquíFoucault analizó la representación a partir del orden por medio de tres ciencias: la gramática general, la historia natural y el análisis de las riquezas. En reemplazo de la semejanza se halla, pues, la representación: el lenguaje entendió la realidad por medio de las representaciones. ¿Cómo lo hace? Por medio de la gramática, la historia natural y las riquezas.

Ahora bien, en este periodo de la época Clásica Foucault analizó la gramática general, la historia natural y el análisis de las riquezas, pensando en que las realidades sensibles son figuradas; estas ciencias son la figura del mundo sensible. De esto se deduce que, aun bajo las mismas figuras del mundo, el discurso pretendió siempre 
ser ordenado. De este modo, Foucault prefiguró lo que después llamará un discurso del orden. Ahora bien, ¿es el hombre quien ordena el saber de este periodo?; aún la figura del hombre vive en la sombra. Aquí el hombre ingresa en una posición ambigua, en el sentido de que es objeto de un saber y, a la vez, sujeto que conoce. La ambigüedad radica, justamente, en que el hombre sea el punto crucial y central de toda reflexión y, sobre todo, sea el autor. Estamos aún muy lejos de que el hombre sea el protagonista capital de este asunto: "es la reivindicación, para el hombre, del derecho a ser sujeto y objeto de la filosofía y del saber que lleva consigo mismo el hombre" (Aaron y Foucault, 2008, p. 29).

Juliette y Justine, de Sade, al igual que El Quijote en la época del Renacimiento, cierra un periodo y abre otro; cerró la época clásica y abrió la modernidad. Ambas obras tuvieron la función de obras bisagras. El discurso de Sade lleva hasta sus últimas consecuencias la representación; ¿en qué sentido lo hizo? Los escritos de Sade que describen la brutalidad y el libertinaje de sus personajes no pretendieron representar los actos de un mundo imaginario, sino superarlos: con los actos de sus libertinos se esconde, por un lado, la destrucción de la escritura por sí misma, y, por otro lado, la destrucción del hombre. Dicha destrucción fue la significación plena del intento de superar la representación. Las letras sadeanas llamaron las cosas por su nombre; no faltó, por lo tanto, seguir las líneas trazadas por la representación; bastó con lo que escribió Sade acerca del hombre; poco a poco se sintió la destrucción de la propia representación, o al menos su consumación, "de manera que el gran relato de la vida de Juliette despliega, a lo largo de los deseos, de las violencias, de las salvajadas y de la muerte, el cuadro centelleante de la representación" (Foucault, 1968, p. 208). No faltó representar con las palabras la imaginación del hombre y su relación con la realidad; la escritura, al estilo sadeano, ofuscó la representación y habló de esa manera de la realidad sin tapujos.

El siglo XIX, el siglo de la episteme moderna, se aferró a la historia de manera que sustituyó el orden, esa noción que estuvo vigente en las dos épocas anteriores. En la época moderna, o en la episteme moderna, las ciencias adquieren nuevas características y abren así el paso a la cientificidad rigurosa: "el esfuerzo por fundamentarlos filosóficamente y justificarlos desde un punto de vista formal; la tentativa de purificar, formalizar y, quizá, matematizar, los dominios de la economía, de la biología y, por último, de la lingüística misma" (Foucault, 1968, p. 241).

La episteme de la época moderna definió el nacimiento del hombre. El hombre eterno no existió desde los inicios de la humanidad; no es el hombre el que siempre 
habitaba las ciencias humanas sino, todo lo contrario, para Foucault fue la episteme moderna la que hizo del hombre un objeto definitivo. Lo más característico de esta episteme fue el descubrimiento de una sombra que vivió en el anonimato, que estuvo lejos de todo antropologismo, que detrás de cada ciencia siempre existió y existirá un espacio inconsciente, una raíz desconocida. ¿Cuál es el tema de esta episteme? La aparición del inconsciente del saber. El crecimiento progresivo de la historia es una mentira, es la "mentira más conocida pero menos reconocida, afirmar que la continuidad misma del movimiento de la razón desde el Renacimiento no es más que una ilusión de óptica” (Burgelin et al., 1970, p. 53).

Luego de analizar las líneas trazadas que giran en torno a las epistemes pudimos percatarnos de los quiebres y las rupturas que se dieron en siglos anteriores con respecto a la aparición de un saber; sin embargo, el desenvolvimiento de los discursos es una cuestión histórica. En este momento debemos dirigir la investigación alrededor de la cuestión acerca de la discontinuidad como rasgo de la historia. ¿Es posible que el análisis histórico de Foucault esté fundado en la discontinuidad? ¿Es la discontinuidad un rasgo de la historia misma y de qué manera revela el movimiento de las epistemes? Esta será la cuestión que ahora pretendo analizar.

\section{LA DISCONTINUIDAD COMO RASGO DE LA HISTORIA}

Debo, pues, para avanzar en este trabajo, delimitar un tipo de preguntas que quiero responder. ¿Cómo funciona la discontinuidad en el análisis histórico?, ¿cómo trabaja en la relación de un periodo a otro? y, en definitiva, ¿cómo estudia ese objeto de estudio en Las palabras y las cosas: la historia de los discursos de saber?

Foucault, fundamentalmente, no describe el paso o qué sucede para que de una episteme se pase a otra. $\mathrm{O}$, ¿cómo se dan esos procesos de saltos? La arqueología no ofrece la explicación de esos procesos (Cfr. Burgelin et al., 1970). Pero, ¿qué separa un periodo de otro si no la misma episteme? Foucault explica la episteme como un espacio de dispersión: "un juego simultáneo de remanencias específicas" (Aaron y Foucault, 2008, p. 50). Es posible ver con más claridad la diferencia de un periodo a otro, en donde una episteme puede cambiar o mutarse, pero no por qué se dan de ese modo dichos cambios.

Entonces, ¿por qué nos inclinamos a pensar que Foucault es un discontinuista? Alrededor delaño 1969, Foucault tuvo una entrevista con el sociólogo Raymond 
Aron, quien lanzó la siguiente pregunta: "¿en qué estructura de pensamiento, o en qué episteme, se encuentra usted?'. Foucault responde:

En primer término, la discontinuidad. La discontinuidad es, por supuesto, un problema muy grave, y bien sabe usted que es un problema que se les plantea a los historiadores. El problema de la periodización se plantea en la historia económica, en la historia social. No hay razón para que los historiadores de las ideas estén retrasados desde el punto de vista metodológico con respecto a los historiadores de la economía o de las sociedades. En el campo de las ideas se plantean problemas de periodizaciones y esta periodización es relativa al nivel en el que nos colocamos y al objeto que se elige.... Tengo la vanidad de haber establecido, creo que por primera vez, la continuidad sincrónica, las relaciones de simultaneidad que había entre campos epistemológicos tan diferentes como el estudio del lenguaje, el estudio de la economía y el estudio de la biología. Compré una discontinuidad al precio de una continuidad o a la inversa, como usted prefiera (Aaron y Foucault, 2008, p. 22).

La opinión de Foucault, dada a Aaron, sirve de manera directa para comprender la relación historia-discontinuidad. Repito mi pregunta anterior: ¿por qué me inclino a pensar que Foucault es un discontinuista? La respuesta es clara: Foucault se encuentra en la estructura del pensamiento de la discontinuidad; sin embargo, como él mismo lo reconoce, la discontinuidad es un problema; un problema que se plantea fundamentalmente a los historiadores, ¿en qué sentido? Los historiadores, por lo general, han tratado de borrar, de olvidar la discontinuidad, en cuanto que ellos, al describir los cortes históricos y tratar de esa manera la relación de un periodo a otro, piensan que existe una relación continua entre estos, omiten que exista un espacio y una ruptura carente de relación. El intento de los historiadores, por lo tanto, es borrar la discontinuidad, mostrar, por el contrario, que existe una lógica interna que recorre de un periodo necesariamente al siguiente; pretenden superar la discontinuidad determinando una continuidad subyacente. Ahora bien, ¿a qué se refiere Foucault al decir continuidad sincrónica?, ¿cómo se entiende su afirmación de que compré una discontinuidad al precio de una continuidad? Para responder ambas cuestiones debemos remitirnos al análisis de la episteme que hemos descrito.

En la episteme moderna, por ejemplo, Foucault describe el desenvolvimiento de la biología, la economía, etc. A primera vista, estas ciencias no tienen relación 
alguna una con otra; sin embargo, el hallazgo de Foucault, su continuidad sincrónica, es haber determinado que entre estas ciencias es posible situar alguna relación en la manera en que producen conocimientos; es decir, la manera o la forma como produce el conocimiento la biología se asemeja a la manera como es producida por la economía: entre estas ciencias existen reglas, quizá puentes, que actúan, según Foucault, de manera invisible. Por otro lado, esta continuidad interna que aparece en una episteme a raíz de las distintas ciencias y sus respectivas y peculiares relaciones son distintas y totalmente diferentes si son comparadas entre una episteme y otra: existe una radical discontinuidad en la manera de producir conocimientos entre las tres epistemes del siglo XVI al XIX. En ese sentido, ¿puede la discontinuidad expresarse claramente alejando las incomprensiones hasta ahora trazadas?, ¿es posible resolver el enigma acerca de qué hace que brote una brecha en el suelo de la historia dando paso a la sucesión, a la irrupción de lo desconocido?

El eje central de la historia que se halla descrita en Las palabras y las cosas, podríamos decir, tiene dos vectores: el empírico y el positivista; entiéndase empírico como las prácticas a partir de los discursos de saber, es decir, todos los ejercicios empíricos elaborados que toman como base el discurso; el lado positivista, por su parte, describe las teorizaciones que el mismo libro entreteje a partir de los conceptos situados en la historia. En ese sentido, la historia descrita por Foucault es el intento de inscribir en la historia, en el devenir histórico, las diversas formas de aparición de las fórmulas empíricas o prácticas y la relación que tienen con sus respectivas teorizaciones, y entre ellas todas las mutaciones que han sufrido en esos movimientos analizando su respectivo a priori histórico.

Ahora bien, para llevar a cabo su análisis histórico, Foucault delimita su espacio de análisis: describe las apariciones de los distintos saberes desde el siglo XVI, los cuales se desplegaron hasta los tres dominios que están representados en el trabajo, la vida y el lenguaje. Su análisis puntualiza, entonces, el lenguaje con el que ha sido posible comprender "el lenguaje tal como era hablado, los seres naturales tal como eran percibidos" (1968, p. 7), y la formación en que nació dicho lenguaje, o dichos lenguajes, formados por medio de códigos, todos ellos a partir del orden. Por consiguiente, entre los saberes que se sitúan a partir del siglo xvı y en la formación del lenguaje debe existir un código de saber, una posible sistematicidad que regula todo nacimiento, que regula el nacimiento de las empiricidades y de las positividades.

En ese sentido, Las palabras y las cosas busca encontrar el punto de inicio en el que nacieron el lenguaje, los discursos y las prácticas o formas empíricas, de tal 
manera que la historia sería la identificación de un modo o tipo de orden siempre azaroso en el que están sujetas las praxis: determinar el modo peculiar de su principio de existencia, "deben sus leyes los cambios, su regularidad los seres vivos, su encadenamiento y su valor representativo las palabras" (Foucault, 1968, p. 7). En otras palabras, según el comentarista Morey, se trata de buscar y seguir los pasos de las mutaciones "de lo empírico en el campo empírico" y "de lo teórico en el campo teórico" (Morey, 1983, p. 231) o, como lo entiende Descombes, "[Foucault] busca la evolución de los conceptos y del pensamiento en los documentos que son los diferentes estados de los diferentes saberes" (1982, p. 138). Llevar a cabo un análisis de lo que de manera visiblemente clandestina está oculto; sobre todo analizar la existencia de una idea de orden que ha llevado a organizar y ordenar todo ello: esa es la tarea de Foucault, como arqueólogo.

¿Qué hace entonces el arqueólogo ante la historia? Su tarea consiste en explicar la manera como durante un determinado tiempo -siglos XVI-XIX-fueron ordenados los conocimientos, cayendo de esa manera en el orden los discursos: sus variaciones, y a partir de ello la manera cómo llegaron a dar paso a saberes diferentes (del análisis del lenguaje se llegó a la lingüística), cuyos análisis están marcados por las condiciones históricas de posibilidad que han hecho posible su nacimiento. En todo ello, el arqueólogo tiene otra tarea más importante: las apariciones, las novedades, los cambios de un tema a otro no deben ser descritos en sus respectivos análisis históricos, es decir, el arqueólogo no elabora un esquema en donde cada aparición de un discurso siga al precedente, no es una historia de las apariciones discursivas de manera sistemática, ya sean continuas o discontinuas; sino que la tarea del arqueólogo consiste en identificar los a priori históricos, gracias a los cuales "pudieron aparecer las ideas, se constituyeron las ciencias, las experiencias pudieron reflexionarse en filosofías, se formaron las racionalidades, quizá, para pronto soltarse y desaparecer" (1968, p. 7). Pues bien, lo que hace el arqueólogo es una suerte de excavación de un campo epistemológico, de una episteme que, analizando sus condiciones de posibilidad, saque a la luz las razones y motivos por los cuales ella misma se ha formado de esa manera y no de otra.

Entonces, lo que Foucault evita es el análisis lineal de los saberes (como lo presentan las ciencias o la historia convencional), en donde la historia de un saber sea descrita de manera creciente y movida hacia su paulatina perfección, asumiendo como consecuencia de lo anterior un telos último. A lo que le llevan sus intereses como arqueólogo, en cambio, es al análisis de la profundidad de los espacios epistémicos, siempre ordenados en cuanto que así fueron constituidos en una episteme, que 
rigen y condicionan los cambios, en los discursos teóricos como prácticos, de los discursos o saberes. En ese sentido, es de esa manera como Foucault demuestra su alejamiento respecto a la lógica de los análisis históricos convencionales. Entonces, la historia no es histórica, no es devenir continuo, es, al contrario, una historia sin telos, sin tiempo lineal (Cfr. Descombes, 1982); para el pensador francés es una historia que describe los espacios del saber, las configuraciones de determinadas epistemes, pero no las vincula en un proceso global y unidireccional. Entonces, de eso se trata: la arqueología es el resultado de la explicación de las condiciones de posibilidad de un saber.

Las palabras y las cosas realiza, entonces, un análisis de la historia en donde no es posible ver la historia como el despliegue racional de un devenir histórico, pasando del renacimiento y terminando en la modernidad; en cambio, el lugar donde podemos situar la historia descrita en la arqueología de las ciencias humanas sería en el análisis de los espacios, los suelos en donde han nacido los discursos, y de esa manera el arqueólogo traza los límites para identificar los cambios, los altibajos siempre inexplicables a la descripción. Todos los discursos de una época, para Foucault, se deben estudiar "fuera de todo criterio que se refiera a su valor racional o a sus formas objetivas" (1968, p. 7). Los discursos, lejos de ser una descripción coherente de hechos históricos que por sí misma explique el desenvolvimiento de estos, deben ser descritos a la manera de la suma paciente de hechos parciales que no buscan ser universales. Me referiré ahora al tema de la discontinuidad, no solo como rasgo de la historia, sino como el rasgo del método arqueológico.

\section{LA DISCONTINUIDAD COMO RASGO DEL MÉTODO ARQUEOLÓGICO}

¿Cómo trabaja el método arqueológico?, es decir, ¿cómo lleva a cabo sus análisis? y ¿qué caminos sigue cuando pretende describir sus investigaciones acerca de la historia? El libro La arqueología del saber, como muchos estudiosos lo han dicho, es la descripción de la puesta en práctica de las investigaciones llevadas a cabo por Foucault (Castro, 1995; Morey, 1993; Díaz, 1993; Dreyfus, 2001). El pensador francés dice: "[La arqueología] se inscribe en ese campo en el que se manifiestan, se cruzan, se entrelazan y se especifican las cuestiones del ser humano, la conciencia y el sujeto" (1969, pp. 26-27). Entonces, ¿qué pretende la arqueología? Buscar y diagnosticar los diferentes cambios y rupturas por las cuales se han constituido los discursos de saber. J. Francois Bert, por su parte, dice: "el problema consistía para Foucault en saber cómo reagrupar elementos lo 
suficientemente emparentados como para que se esclarecieran mutuamente, pero también lo suficientemente heterogéneos como para permitir la deducción de un sistema de relación válido" (cit. en: Burgelin et al., 1970, p. 29). En ese sentido, el método que expone La arqueología del saber es, de alguna manera, la comparación: comparar elementos comunes y diferentes para, a partir de ello, describir un sistema válido.

Si bien la biología, la economía y la lingüística, aparentemente, no tenían contacto, la arqueología permitía componer un sistema de relación válido, más allá de las aparentes rupturas, simultaneidades o continuidades sincrónicas. Habría que preguntar a Foucault acerca de "la manera en que afirmaba la discontinuidad fundamental entre dos configuraciones de saberes" (R. Aaron y M. Foucault, 2008, p. 44); pues la historia o el análisis histórico que describa o analice la relación de una episteme a otra siempre será una actividad infecunda: las epistemes no tienen relación una junto a la otra. A partir de lo anterior, la distinción sería el segundo elemento del método arqueológico.

Las cosas, el lenguaje, el discurso brotan por símismos a la superficie, existe una “erosión del exterior" (Cfr. Foucault, 1991b, pp. 31-33); es decir, que llegará el momento en que el investigador descubra un tipo de erosión que aparecerá en la superficie, en la historia. No hace falta más tarea que la paciente espera y la acumulación de información acerca del nacimiento y, posteriormente, de los cambios de un discurso, del desenvolvimiento de un discurso entre un periodo y otro; aunque la insistencia caiga en que no sea posible un análisis de manera rígida y seria de dichos cambios: "esta abertura profunda en la capa de las continuidades, si bien debe ser analizada, y debe serlo minuciosamente, no puede ser 'explicada', ni aun recogida en una palabra que se reparte sobre toda la superficie visible del saber y cuyos signos, sacudidas y efectos pueden seguirse paso a paso" (1968, p. 213).

Ahora bien, lancemos la pregunta: ¿de qué manera se configura el método arqueológico frente a una episteme? Digamos: el arqueólogo tendrá que reunir una vasta información de documentos acerca de un periodo; la tarea del arqueólogo será leer y releer todo cuanto a su alcance sea posible: es un erudito en cuanto a los todos los saberes; luego del estudio, inicia el trabajo de selección: reorganiza un determinado tema a partir de la información recolectada. La búsqueda del tema o el enunciado, "acontecimiento extraño, indudablemente (...) porque está ligado por una parte a un gesto de escritura o a la articulación de una palabra" (1969, p. 46), que está entre sus intereses arqueológicos. Luego se llevaría a cabo el análisis 
de dicha división: qué temas emergen en cuanto a un grado de importancia: "lo que hace posible el conjunto de la episteme (...) es, ante todo, la relación con un conocimiento del orden" (1968, p. 78). Se trata, en cuestión, de "buscar un fundamento en esas figuras del saber en las que participan, y que constituyen la novedad radical de la época del hombre: las figuras que aparecen y las que inician su crepúsculo en la historia” (1969, p. 116). ¿Qué sucede con un tema cuando está presente de manera gradual pero no primordial? Aquí la tarea del arqueólogo es señalar la diferencia o subordinación. Existen temas o discursos que han pasado de un lado a otro en la historia, no por ser discursos que no se relacionen, sino porque existían otros discursos antes que ellos, o existían los primeros en menor grado (Cfr. Foucault, 1969, pp. 50-64). Por todo lo desarrollado líneas arriba, el tercer momento del método arqueológico consistiría en el análisis de los documentos de manera libre y fortuita.

Continuemos las referencias en torno a la discontinuidad como rasgo del método arqueológico que se nos ofrece en La arqueología del saber a la luz de lo anteriormente mencionado. En primer lugar, Foucault ha tomado la historia de las ciencias humanas como un tema arqueológico, y los discursos de saber, en una perspectiva histórica a partir de la arqueología, se trata de "un método de análisis histórico liberado del tema antropológico" (1969, p. 26). De la episteme renacentista a la episteme moderna, el lenguaje ha cambiado, ha mutado, de tal manera que es pertinente decir que cada época tuvo una noción, más o menos clara, de lo que le era posible hablar: la idea que se tuvo del lenguaje y en la que se construyó un saber, "hace emerger en su pureza el espacio donde se despliegan los acontecimientos discursivos (...) para describir, dentro y fuera de él, los juegos de relaciones" (1969, p. 41). Cabe aquí destacar que desde el renacimiento hasta la modernidad el sentido interno con que se analizó la historia fue el de ordenar los saberes: en el renacimiento, como vimos, por la semejanza (para no confundir que una cosa tenga semejanza con otra, este tiempo define cuatro modos: la conveniencia, la emulación, la analogía y la simpatía); en la época clásica se ordenaron los saberes mediante la representación, y la modernidad manejará el espacio inconsciente, el espacio del saber, donde el lenguaje no dice nada de sí mismo, sino que su saber está orientado hacia una ciencia: todo nuestro saber está determinado por un lenguaje que lo controla, lo educa, lo rige y lo determina.

Siempre se mantuvo la idea, y quizá sea el reproche hacia Foucault, de que no explica su trabajo, específicamente en lo que se refiere a su método. La prueba más reiterativa es la falta de información acerca de cómo explicar el trance, el 
cambio, la ruptura de una episteme a otra. El círculo de epistemología dirigió en 1968 una carta a Foucault como respuesta a la publicación de sus libros y a las incomprensiones que ellos generaban. “¿Cuál es el motor que transforma a una configuración en otra?" (Burgelin et al., 1970, p. 219), fue la pregunta de $E l$ círculo de epistemología. Las respuestas del pensador francés al círculo de epistemología causaron asombro a todos esos lectores, en cuanto que se convertirían en las principales líneas de su próximo libro: La arqueología del saber. Por ahora detallaré brevemente algunas de las respuestas que dio el pensador francés, para pasar a denominar la cuestión del método arqueológico frente a la historia y la discontinuidad.

Evidentemente, entre la configuración de un periodo y otra existe una discontinuidad. La cuestión de la discontinuidad en Foucault sufrió un cambio de estatus, una profunda mutación frente a la concepción que la historia tenía de ella. Para la historia, la cuestión de la discontinuidad era aquello que el historiador tenía la obligación de hacer desaparecer de la historia, y podía muy bien explicarse tal tarea a partir de dos palabras: de lo dado y lo impensable. En primer lugar, todas las cuestiones acerca de las instituciones, las teorías y las prácticas reflejan lo dado; todo este conjunto que es posible percibir, que es posible estudiar, analizar y, sobre todo, registrar; lo anterior se refleja en la sociedad, en las culturas, en el sentido de que son teorías y prácticas al alcance del historiador. En segundo lugar, las teorías y las prácticas que se dan de la misma manera en la sociedad y en la cultura, pero que corresponden a ser anónimas, subjetivas e infundadas reflejan lo impensable.

El historiador frente a ello debe, como dijimos, borrar lo impensable de la historia para que de esa manera no salga a la superficie, y no pueda así aparecer; en efecto, para la historia lo dado es lo continuo, y lo impensable es lo discontinuo; en ese sentido, por debajo de la continuidad circulaba la discontinuidad de manera ilegal. El pensador francés se alejará de esta concepción de la discontinuidad, para darle otro sentido; dirá Foucault: "la discontinuidad era ese estigma del desperdigamiento temporal que el historiador estaba encargado de suprimir de la historia" (1969, p. 223). Pero todo fue cambiando en esa mirada, y ello trajo que la discontinuidad sea el elemento clave, importante, elemental para llevar a cabo un análisis histórico. Foucault, por lo que acabo de decir, siempre mostró un interés acerca de la discontinuidad como elemento importante.

En primer lugar, imbuido en la mirada arqueológica, el historiador debe delimitar su campo de análisis, diferenciar los posibles niveles de análisis y, sobre todo, 
determinar qué periodo es el que le interesa. En segundo lugar, lo que le interesa al historiador es descubrir todos los posibles límites del desenvolvimiento del tema que se ha propuesto analizar, "los límites de una oscilación, el umbral del funcionamiento" (Burgelin et al., 1970, p. 223). Finalmente, en tercer lugar, el rol de la discontinuidad no hace de navaja, cortando por el medio y separando dos saberes dentro de una esfera positiva, al contrario, en cada periodo, en cada dominio, en cada nivel adopta una forma y una función. En definitiva, la discontinuidad es a la vez instrumento y objeto de investigación; Foucault explica lo anterior así: "[la discontinuidad] permite individualizar los dominios, pero sólo se la puede establecer comparando a estos dominios; no rompe unidades, sino para establecer otras nuevas; escande series y desdobla niveles; $y,(. .$.$) no es$ simplemente un concepto presente en el discurso del historiador, sino que éste, en secreto, lo supone" (Foucault, 2013, p. 227).

La discontinuidad, entonces, va más allá de ser una simple brecha que separa dos espacios o momentos históricos; se sitúa en cada separación de hechos o sucesos, analiza aquello que la desvincula con el precedente; su tarea es la de la comparación, "sólo se la puede establecer comparando". Toda la tarea del historiador, entonces, es una tarea discontinua implícitamente: "el uso reglado de la discontinuidad para el análisis de las series temporales" (Foucault, 1969, p. 278). Hemos venido describiendo la continuidad y la discontinuidad como rasgos simultáneos de la historia, ¿será posible ver la continuidad como lo visible y la discontinuidad como lo invisible de esa historia?, ¿será extravagante insinuar que la continuidad puede ser analizada como lo consciente y la discontinuidad como lo inconsciente?

En una entrevista titulada "Verdad y poder" (Verité et pouvoir), dice el pensador francés acerca de la discontinuidad:

Una edición del "Petit Larousse" que acaba de aparecer dice: "Foucault: filósofo que funda su teoría de la historia en la discontinuidad". Esto me dejó boquiabierto. Sin duda no me expliqué suficientemente en Las palabras y las cosas, pese a que hablé mucho sobre ello. Me parece que en ciertas formas empíricas del saber, como la biología, la economía política, la psiquiatría, la medicina, etc., el ritmo de las transformaciones no obedecía a los esquemas dulces y continuistas del desarrollo, tal y como habitualmente se piensa (1999b, p. 43).

Según Canguilhem, el aporte del trabajo de Foucault fue descubrir el inconsciente de los discursos de Occidente, el cual estaba detrás de todo el saber de la sociedad 
moderna: "Pero sus respuestas mordaces a objeciones a menudo rutinarias, no sólo constituían una defensa, sino también, las más de las veces, la iluminación fulminante de sus incursiones en el inconsciente de los conocimientos, de sus preguntas y respuestas" (Burgelin et al., 1970, p. 77). La historia, en ese sentido, es el proceso consciente: las continuidades no parceladas, los largos encadenamientos, los análisis prolongados de los hombres, de sus palabras y de sus gestos. Y la discontinuidad es el proceso inconsciente: estudia las razones siempre ocultas, oscuras, por las cuales es posible mirar todo ese espacio, todo ese horizonte continuo, todo ese amanecer de la consciencia, toda la historia totalizadora que a partir de ella emana. La sospecha del inconsciente ante la conciencia se confirma una vez más: "cuando la historia no ofrece bastante seguridad, es al devenir del pensamiento, de los conocimientos, del saber, es al devenir de una conciencia siempre próxima de sí misma (...) que se pide salvar lo que debe ser salvado" (Foucault, 2012, p. 226).

\section{Conclusión}

Este trabajo de investigación siguió el recorrido intelectual del pensador francés a partir de una lectura en clave discontinua. Pasando del análisis acerca de la historia, como objeto de estudio, y de la arqueología, como método de estudio, en el periodo caracterizado como arqueológico en el pensamiento de Foucault, he trazado las líneas claves que sostienen la afirmación de que Foucault pensó su trayecto intelectual a partir de una idea inherente marcada constantemente por el tema de la discontinuidad; por lo tanto, el tema de la discontinuidad está consecuentemente latente.

Vimos que el tema de la discontinuidad está situado inseparablemente de la visión de historia del pensador francés, determinando así una manera de entender y llevar a cabo una historiografía; esto tiene sus particularidades. En dicho análisis podemos, como lo hizo Foucault, asignar un nombre a una episteme para referirnos a cierto uso que ella cumplió en un periodo, pero lo que no está al alcance de quien realiza un análisis histórico inspirado en el pensador francés es comprender por qué se producen los diversos cambios, en ocasiones hasta bruscos, entre una episteme y otra. La discontinuidad está presente en la insuficiencia, o imposibilidad, de comprender de qué razones se sirve una episteme para realizar el salto o movimiento hacia la siguiente. Una discontinuidad, por lo tanto, que no prohíbe al historiador plantear las diferencias entre las epistemes, pero sílo deja en un total mutismo al momento de comprender el porqué del cambio de una hacia otra. Por lo tanto, 
podemos afirmar que, en el periodo arqueológico, el tema de la discontinuidad se encuentra ligado a no explicar la separación entre cada episteme, pero respeta, evidentemente, cierta coherencia en el movimiento interno, es decir, no existe ningún tipo de discontinuidad en el juego, funcionamiento o en cómo se llegan a formar los saberes en cada periodo. En suma, una discontinuidad que acentúa aspectos generales. ¿De qué manera? El tema de la discontinuidad ayuda a comprender la manera como han sido ordenados los conocimientos, sus variaciones, dentro de un periodo; como método compara los elementos comunes y diferentes; distingue un saber en cuanto es distinto de otro. Todo lo anterior en el marco siempre de un periodo. Por todo ello, se trata de una discontinuidad que respeta el proceso interno en la formación de saberes dentro de cada periodo, pero que se manifiesta radical en el cambio de un periodo a otro; eso por un lado.

Existe un aporte fundamental, pienso, en la comprensión del tema de la discontinuidad en el periodo arqueológico. Foucault introdujo, entonces, en el análisis de la historia, la discontinuidad como elemento clave para comprender más a fondo la historiografía, que debe emerger a raíz de un conjunto de hechos claros y razones siempre ocultas. Durante bastante tiempo, cierto análisis histórico pretendió eliminar o hacer desaparecer la discontinuidad, pues iba en contra de un interés muy importante para sus realizadores: la continuidad en sus trabajos históricos. El aporte de Foucault consiste en afirmar que no hay necesidad de hacerla desaparecer, pues la historia es el resultado de análisis continuos y discontinuos, conscientes e inconscientes.

Dos son, entonces, los ejes en los que se mueve el tema de la discontinuidad: la imposibilidad de explicar la brecha entre un periodo y otro y, fundamentalmente, la visión positiva de este tema en la historiografía. Mediante el desenvolvimiento hasta aquí tratado podemos asegurar que el tema de la discontinuidad aparece en el marco de su reflexión sobre la historia a partir del método arqueológico, es decir, la discontinuidad constituye una vena por la que recorre gran parte de la reflexión de Foucault en el periodo arqueológico. Cabe abrir una nueva pregunta frente al segundo momento del trabajo de Foucault, es decir, frente al periodo genealógico, antes de dar por concluido este trabajo: ¿qué sucede en ese momento, es decir, el tema de la discontinuidad está presente en el periodo genealógico como estuvo en el periodo arqueológico o se trata quizá de una cuestión marginal? No nos demos por satisfechos. La cuestión anterior exige una pronta respuesta. 


\section{REFERENCIAS}

Aron, R., Foucault, M. (2008). Diálogo: Raymond Aron y Michel Foucault. Buenos Aires: Nueva Visión.

Burgelin, P., Revault, O., Amiot, M. (1970). Análisis de Michel Foucault. Buenos Aires: Tiempo Contemporáneo.

Canguilhem, G. (2005). Ideología y racionalidad en la historia de las ciencias de la vida. Buenos Aires: Amorrortu.

Castro, E. (1995). Pensar a Foucault. Buenos Aires: Biblos.

Castro, E. (2004). El vocabulario de Michel Foucault. Bernal: Universidad Nacional de Quilmes.

Ceballos Garibay, H. (1997). Foucault y el poder. México: Coyoacan S. A.

Descombes, V. (1982). Lo mismo y lo otro. Madrid: Cátedra.

Díaz, E. (1993). Michel Foucault. Los modos de subjetivación. Buenos Aires: Almagesto.

Deleuze, G. (1987). Foucault. Barcelona: Paidós.

Dreyfus, H., Rabinow, P. (2001). Michel Foucault: Más allá del estructuralismo y de la hermenéutica. Nueva Visión: Buenos Aires.

Foucault, M. (1968). Las palabras y las cosas. México: Siglo XXI. (Trad. E. Frost).

Foucault, M. (1969). Arqueología del saber. Madrid: Siglo XXI. (Trad. A. Garzón del Camino).

Foucault, M. (1991b). Saber y verdad. Madrid: La Piqueta. (Ed. y Trad. de J. Varela y F. Álvarez Uría).

Foucault, M. (1999b). Estrategias de poder. Tomo II. Barcelona: Paidós.

Foucault, M. (1983). Contestación al Círculo de Epistemología. En: Terán, O. (compilador). Michel Foucault. El discurso del poder. México: Folios Editora. 
Foucault, M. (2000). Nietzsche, la genealogía, la historia. Madrid: Pre-textos.

Foucault, M. (2012). El poder una bestia magnífica. Buenos Aires: Siglo Veintiuno. (Trad. Horacio Pons).

Le Blanc, G. (2008). El pensamiento de Michel Foucault. Buenos Aires: Amorrortu.

Morey, M. (2014). Lectura de Foucault. Madrid: Taurus.

Revel, J. (2009). Diccionario Foucault. Buenos Aires: Nueva Visión.

Revel, J. (2014). Michel Foucault. Un pensador de la discontinuidad. Buenos Aires: Amorrortu.

Sauquillo, J. (2001). Para leer a Foucault. Madrid: Alianza. 